opportunities for earlier HIV diagnosis are being missed in primary care. We assessed changes in GPs' HIV testing behaviour following an educational intervention using competitive feedback, to improve HIV testing in primary care in Amsterdam.

Methods The educational intervention, open for all Amsterdam GPs, was implemented from 2015 to 2020. The mean annual number of HIV tests per GP from 2011-2019 was calculated using data from diagnostic laboratories for primary care, and stratified by 4-digit postal code (PC4). Questionnaires and semi-structured interviews were conducted to identify perceived barriers and facilitators to HIV testing.

Results In total, 229 GPs (42\%) participated in the educational intervention. Participation varied per PC4 area (median 27\%, IQR 0\%-60\%). At baseline, the mean annual number of HIV tests per GP was similar for participants versus non-participants (26.8 versus 24.7, respectively). The number of tests per GP declined from 2011 to 2014 from 29.5 to 20.7, and increased thereafter to 27.1 in 2019. Testing was highest in PC4 areas with highest HIV prevalence. Qualitative analyses revealed various barriers to HIV testing, including taboo and stigma, a shrinking epidemic, and financial barriers. The use of competitive feedback was perceived as a motivator to improve testing behaviour. Of 59 GPs that completed the questionnaire, $68 \%$ stated the programme provided eye-openers, and $72 \%$ declared it improved their HIV testing behaviour.

Conclusion The observed increase in HIV testing coincided with the implementation of our intervention, but there was marked heterogeneity, with testing seemingly associated with local HIV prevalence. Amsterdam is well on its way towards zero new hiv infections, but it will be challenging to keep GPs engaged in proactive testing to prevent late presentations and missed opportunities for HIV diagnosis in primary care.

\section{P275 PREPPED FOR COVID? EXPLORING THE ASSOCIATION BETWEEN HIV PRE-EXPOSURE PROPHYLAXIS USE AND COVID-19 EXPERIENCE AMONG MSM}

\begin{abstract}
${ }^{1,2} \mathrm{C}$ Pulford ${ }^{*},{ }^{1,2} \mathrm{H}$ Mitchell, ${ }^{2,3} \mathrm{~A}$ Howarth, ${ }^{1,2,3}$ J Saunders, ${ }^{2,3} \mathrm{C}$ Mercer, ${ }^{1,2} \mathrm{G}$ Hughes. ${ }^{1}$ Blood Safety, Hepatitis, STIs and HIV Division, National Infection Service, Public Health England, Colindale, UK; ${ }^{2}$ The National Institute for Health Research Health Protection Research Unit in Blood Borne and Sexually Transmitted Infections at University College London in partnership with Public Health England, London, UK; ${ }^{3}$ Institute for Global Health, University College London, London, UK
\end{abstract}

\subsection{6/sextrans-2021-sti.346}

Background Pre-exposure prophylaxis (PrEP) is highly effective at reducing HIV acquisition. Studies are underway to investigate the effectiveness of HIV antiretrovirals, including Tenofovir-based PrEP, for treating and preventing COVID-19. We investigated the association between HIV-PrEP use and COVID-19 among men who have sex with men in the UK.

Methods Participants completed an online survey (23/06/20$14 / 07 / 20$ ), including men (cis/transgender), transwomen or gender-diverse people reporting sex with another man (cis/ transgender) or non-binary person assigned male at birth. The outcome was COVID-19 experience, defined as reporting a positive test (antigen/antibody) or symptoms of a new continuous cough, high temperature or anosmia following the implementation of UK-wide restrictions ('lockdown' 23/03/20). All participants reporting taking HIV-PrEP since the beginning of the COVID-19 pandemic (12/19) were compared with those who did not. Analysis was performed using logistic regression, adjusting for sociodemographics (age, ethnicity, education, country) and subsequently for behavioural factors during lockdown (relationship status, chem-sex and number of new partners).

Results Altogether, 1,814 (89.9\%) participants reported that they were living without HIV, of whom 253 (14.0\%) reported experiencing COVID-19 (positive test or symptoms). Since December 2019， 410 (22.6\%) participants reported taking HIV-PrEP, with daily use being lower during lockdown (6.2\%) compared with the three months pre-lockdown (14.3\%). HIVPrEP use was positively associated with COVID-19 (crude$\mathrm{OR}=1.51, \quad 95 \% \mathrm{CI}=1.13-2.04, \quad \mathrm{p}=0.006), \quad$ including after adjusting for sociodemographics $(\mathrm{OR}=1.57$, 95\%CI:1.16-2.11, $\mathrm{p}=0.005)$, and after further adjustment for behavioural factors $(\mathrm{OR}=1.51,95 \% \mathrm{CI}: 1.11-2.06, \mathrm{p}<0.01)$.

Conclusion There is a positive association between HIV-PrEP use and COVID-19, independent of number of new partners. Our findings may reflect behaviours that increase COVID-19 exposure amongst HIV-PrEP users that are not captured in our analysis. People may also perceive that HIV-PrEP offers them protection against COVID-19. However, until robust evidence is available, people taking HIV-PrEP should adhere to COVID-19 prevention advice.

\section{P277 HOW DO WE MEASURE UNMET NEED WITHIN SEXUAL AND REPRODUCTIVE HEALTH? A SYSTEMATIC REVIEW}

${ }^{1} \mathrm{D}$ Solomon*, ${ }^{2} \mathrm{M}$ Cabecinha, ${ }^{1} \mathrm{~J}$ Gibbs, ${ }^{3} \mathrm{~F}$ Burns, ${ }^{1} \mathrm{C}$ Sabin. ${ }^{1}$ Institute for Global Health, University College London, London, UK; ${ }^{2}$ Research Department of Primary Care and Population Health, University College London, London, UK; ${ }^{3}$ Royal Free London NHS Foundation Trust, London, UK

\subsection{6/sextrans-2021-sti.347}

Background Addressing health inequality with sexual and reproductive health requires an understanding of unmet need within a range of populations. This review examined the methods and definitions that have been used to measure unmet need, and the populations most frequently assessed.

Methods Five databases (PubMed, Web of Science, Scopus, CINAHL and HMIC) were searched for studies that described quantitative measurement of unmet need within sexual and/or reproductive health between 2010 to 2020. A narrative synthesis was then undertaken to ascertain themes within the literature.

Results The database search yielded 18539 papers; 145 papers were included after screening.

128 studies assessed unmet reproductive health need, of which 94 were analyses of trends among women living in low/lower-middle income countries; 121 used cross-sectional data, with only seven analyses being longitudinal.

Twelve studies analysed unmet sexual health need, of which nine focused on high and upper-middle income populations. All twelve used cross-sectional analyses.

The remaining five studies examined unmet need for a combination of sexual and reproductive health services, all among populations from upper-middle or high income countries and all being cross-sectional analyses.

138 of the papers were analyses of questionnaire data, five used reviews of medical records, one compared demand before and after an intervention, and one used modelling techniques.

113 studies used the DHS definition of unmet need; no other standardised definition was used among the remaining papers. 
Discussion There is a significant focus on unmet need for contraception among women in low income countries within the published literature, leaving considerable evidence gaps in relation to unmet need within sexual health generally and among men in particular, and unmet reproductive health need in high income settings. Additionally, using an increased range of data collection methods, analyses and definitions of unmet need would enable better understanding of health inequality in this area.

\section{P281 EXPLAINING EXPERIENCES OF ACCELERATED PARTNER THERAPY PARTNER NOTIFICATION FOR PEOPLE WITH CHLAMYDIA IN THE LUSTRUM RANDOMISED CONTROL TRIAL: PROCESS EVALUATION}

\begin{abstract}
${ }^{1} \mathrm{~F}$ Mapp*, ${ }^{2,3} \mathrm{C}$ Estcourt, ${ }^{4} \mathrm{~J}$ Cassell, ${ }^{2} \mathrm{~J}$ MacQueen, ${ }^{1} \mathrm{~A}$ Howarth, ${ }^{5} \mathrm{~S}$ Brice, ${ }^{5} \mathrm{~A}$ Comer, ${ }^{6} \mathrm{M}$ Symonds, ${ }^{3,7,8} \mathrm{R}$ Nandwani, ${ }^{1} \mathrm{M}$ Woode Owusu, ${ }^{1,9} \mathrm{~J}$ Saunders, ${ }^{1} \mathrm{C}$ Mercer, ${ }^{1} \mathrm{O}$ Stirrup, ${ }^{1} \mathrm{~A}$ Copas, ${ }^{10} \mathrm{~N}$ Low, ${ }^{11} \mathrm{~T}$ Roberts, ${ }^{2} \mathrm{M}$ Pothoulaki, ${ }^{1} \mathrm{~A}$ Tostevin, ${ }^{10} \mathrm{C}$ Althaus, ${ }^{11} \mathrm{C}$ Ogwulu, ${ }^{1,12}$ S Wayal, ${ }^{1} \mathrm{~A}$ Johnson, ${ }^{13} \mathrm{P}$ Flowers. ${ }^{1}$ University College London, London, UK; ${ }^{2}$ Glasgow Caledonian University, Glasgow, UK; ${ }^{3}$ NHS Greater Glasgow and Clyde, Glasgow, UK; ${ }^{4}$ Brighton and Sussex Medical School, Brighton, UK; ${ }^{5}$ Barts Health NHS Trust, London, UK; ${ }^{6}$ Western Sussex Hospitals NHS Foundation Trust, Worthing, UK; ${ }^{7}$ University of Glasgow, Glasgow, UK; ${ }^{8}$ Public Health Scotland, Edinburgh, UK; ${ }^{9}$ Public Health England, London, UK; ${ }^{10}$ University of Bern, Bern, Switzerland; ${ }^{11}$ University of Birmingham, Birmingham, UK; ${ }^{12}$ Development Media International CIC, London, UK; ${ }^{13}$ University of Strathclyde, Glasgow, UK
\end{abstract}

\subsection{6/sextrans-2021-sti.348}

Background Accelerated partner therapy (APT) is a partner notification (PN) method whereby healthcare professionals assess sex partners by telephone, then send or give the index patient antibiotics and self-sampling kits for their sex partner (s). APT was implemented within a cluster cross-over randomised control trial in 17 sexual health clinics in Britain (2018-2019, ISRCTN Reference 15996256). We conducted an integral process evaluation to help explain experiences of using APT.

Methods Focus groups and telephone interviews with 34 healthcare professionals who delivered APT, and telephone interviews with 15 index patients and 17 sex partners who chose APT. Topic guides focussed on how APT was implemented and overall APT experiences. Data were analysed deductively using a bespoke framework derived from initial conceptualisations of APT, and key trial findings.

Results Low uptake of APT was largely because index patients felt it was only suitable for certain types of sex partner. APT was considered best suited to established relationships and not appropriate for relationships with lower emotional connection. However, APT was not always offered by healthcare professionals and many sex partners attended clinic with index patients when they attended for treatment. Nevertheless, those who chose APT felt it worked better than existing options and helped partners overcome barriers to face-to-face care. Most sex partners received APT packs directly from the index patient within a day of consultation; some prioritised taking treatment over self-sampling. Some sex partners reported difficulties in blood sampling (finger-prick) resulting in fewer HIV and syphilis samples being returned than chlamydia and gonorrhoea (urine/vulvo-vaginal swab). Some sex partners did not value testing for infections other than chlamydia/gonorrhoea. Conclusions APT benefits established sexual partnerships with greater emotional connection, by providing treatment rapidly and overcoming barriers to face-to-face care. Targeting of APT combined with interventions to increase sex partner return of self-samples are needed.

\section{P283 DIFFERENCES IN RISK PERCEPTION AND RISK BEHAVIOR BETWEEN PREP-USING AND NON-PREP- USING MSM IN THE NETHERLANDS}

\begin{abstract}
${ }^{1} \mathrm{~S}$ Diexer ${ }^{*},{ }^{1} \mathrm{~A}$ Teslya, ${ }^{2,3} \mathrm{D}$ Rojas Castro, ${ }^{2} \mathrm{R}$ Delabre, ${ }^{4} \mathrm{~K}$ Jonas, ${ }^{1} \mathrm{M}$ Kretzschmar. ${ }^{1} J u l i u s$ Center for Health Sciences and Primary Care, University Medical Center Utrecht, Utrecht University, Utrecht, The Netherlands; ${ }^{2}$ Community-Based Research Laboratory, Coalition PLUS, Pantin, France; ${ }^{3}$ Aix Marseille Univ, INSERM, IRD, SESSTIM, Sciences Economiques and Sociales de la Santé and Traitement de I'IInformation Médicale, Marseille, France; ${ }^{4}$ Department of Work and Social Psychology, Faculty of Psychology and Neuroscience, Maastricht University, Maastricht, The Netherlands
\end{abstract}

\subsection{6/sextrans-2021-sti.349}

Background Pre-exposure prophylaxis (PrEP) uptake has remained low among men who have sex with men (MSM) eligible for PrEP in the Netherlands, which could partly be due to stigma associated with taking PrEP. Perceptions about PrEP users (e.g., PrEP users have an increased rate of (condomless) sexual encounters) may modify mixing behavior and subsequently affect dynamics of HIV and STIs. We investigated whether HIV and STI risk perception, risk behavior, and partner selection depend on PrEP usage.

Methods We analyzed sexual behavior and PrEP data from 426 HIV-negative MSM who participated in the Flash! PrEP in Europe survey in the Netherlands in 2016. We distinguished between current PrEP users $(n=29)$, men interested in $\operatorname{PrEP}(n=267)$, and men not interested in PrEP $(n=130)$. We tested differences between these 3 groups in risk perception and sexual behavior variables using ANOVA and Chi-squared test.

Results Mean age of participants was 42 yrs (range $=18-75$ ); $85 \%$ were born in the Netherlands. PrEP users and men not interested in PrEP consider their risk of acquiring HIV low, while men interested in PrEP consider their risk higher $(p<0.001)$. Perceived risk of getting infected with STIs is higher for PrEP users and men interested in PrEP $(p<0.001)$. PrEP users have significantly more sex partners (mean $=53$, $(\mathrm{SD}=95)$ vs. $13(20)$ vs. 3 (6), p<0.001), and more condomless sex $(\mathrm{p}<0.001)$. PrEP users and men interested in PrEP are less likely to refuse to have sex and more likely to have condomless sex with a PrEP user $(\mathrm{p}<0.001)$.

Conclusion PrEP users engage in sexual behavior with higher risk of STI acquisition than non-PrEP users. Men not interested in PrEP are less open for sexual encounters with PrEP users than men in other groups. Therefore, perceptions of PrEP users and non-PrEP users may change mixing patterns and thus STI dynamics.

\section{P286 QUALITATIVE FINDINGS FROM NATSAL-COVID: EXPLORING DIFFICULTIES AND DISTRESS WITHIN ESTABLISHED RELATIONSHIPS DURING COVID-19 PANDEMIC}

${ }^{1} \mathrm{R}$ Boso Perez ${ }^{*},{ }^{1} \mathrm{~K}$ Maxwell, ${ }^{2} \mathrm{D}$ Reid, ${ }^{3} \mathrm{C}$ Tanton, ${ }^{3} \mathrm{~W}$ Macdowall, ${ }^{3} \mathrm{C}$ Bonell, ${ }^{2} \mathrm{~S} C$ Clifton, ${ }^{2} \mathrm{P}$ Sonnenberg, ${ }^{2} \mathrm{C}$ Mercer, ${ }^{2} \mathrm{~N}$ Field, ${ }^{1} \mathrm{~K}$ Mitchell. ${ }^{1}$ University of Glasgow, Glasgow, UK; ${ }^{2}$ University College London, London, UK; ${ }^{3}$ London School of Hygiene and Tropical Medicine, London, UK

10.1136/sextrans-2021-sti.350 